\title{
Internal Model Principle for Measurement Feedback Problem
}

\author{
Zhiyong Chen
}

\begin{abstract}
While the disturbances in actuators are well handled by the output regulation theory (especially, called input disturbance suppression problem), there are rare results to deal with those in sensors. When disturbances enter sensors, the plant states cannot be precisely measured, and the state feedback control laws are not implementable. Instead, the measurement feedback control laws become interesting. This paper gives a new mechanism using the internal model principle for designing measurement feedback control laws to solve the robust regulation problem. The proposed controllers have the robustness with respect to sensor disturbances. The mechanism derives from the output regulation theory, and puts this theory to a new stage.
\end{abstract}

\section{INTRODUCTION}

Ubiquitous disturbances exist in a control loop, and control engineers are interested in designing a feedback control law to make the closed-loop system display the desired behavior in presence of these disturbances. A controller's ability of rejecting disturbances is called its robustness property, which is one of the most important issues in control theory and applications. According to the location where the disturbances exist in the control loop, the disturbances can be classified as plant disturbance, actuator disturbance, and sensor disturbance. And these disturbances can be dealt with by different approaches in literatures.

Since 1970s, the output regulation theory has been well developed for disturbance rejection problem, first for the class of linear system (see [5], [6], etc.) The paper [11] by Isidori and Byrnes is the cornerstone of the nonlinear output regulation theory. And some recent development of the nonlinear output regulation theory and applications can be found in the monograph [9] by Huang. A main outcome of the efforts on robust output regulation problem is the internal model principle. However, the existing formulation of output regulation problem only accommodates plant disturbance and/or actuator disturbance.

When disturbances enter sensors, the disturbance rejection problem becomes much more complicated. Feedback is the fundamental philosophy of closed-loop control methodologies. To achieve the global stabilization or regulation of a nonlinear system, most controllers are in the form of state (or partial state) feedback. In fact, output feedback controllers only exist for some particular nonlinear systems with certain linear growth constrains [13]. However, when the disturbances appear in sensors, the states cannot be

Z. Chen is with the School of Electrical Engineering and Computer Science, The University of Newcastle, NSW 2308, Australia zhiyong. chen@newcastle.edu.au

The work described in this paper was partially supported by the Early Career Researcher Grant G0187304. precisely measured. As a result, the state feedback controllers cannot be implemented. Instead, we appeal to the so-called measurement feedback control. In literatures, there is no general method for designing measurement feedback control because of the difficulty shown by a counter example given in [7] that for some nonlinear systems, there does not exist any continuous state feedback controller so that bounded sensor disturbances produce bounded states. Nevertheless, some investigation has been conducted on measurement feedback control in [8], [12], [14] and references therein. In particular, in [12], the small gain theorem is used to design a partial state feedback controller such that the closed-loop system is input-to-state stable with the sensor disturbance as input. To the best of the author's knowledge, there is no general constructive approach for nonlinear controllers which can completely suppress the sensor disturbances, that is, to drive all states of the plant to the equilibrium point in presence of sensor disturbances, rather than to achieve the input-to-state ability with sensor disturbance as input. Actually, as we will discuss in the next section, without precise state feedback, it is almost impossible to drive all states of the plant to the equilibrium point. Nevertheless, there is a possibility to achieve this objective if we have a certain structural information of the disturbances. In this paper, we investigate this possibility using the internal model principle.

The main control objective is to drive all plant states to the equilibrium point using measurement feedback, called stabilization or regulation problem. To achieve this objective, we first design an internal model working as an estimator for the so-called disturbance generator. Then, an augmented error system is obtained by performing a certain coordinate transformation on the system composed of the plant and the internal model. We further prove that the solvability of the measurement feedback stabilization or regulation problem of the original system is implied by that of the state feedback stabilization or regulation problem of the augmented error system. Finally, we solve the resulted state feedback stabilization or regulation problem on case by case basis. Technically, the conversion is motivated by the recent development on the output regulation theory [3], [4], [10], etc. The contribution of this paper is two fold. First, we provide a new technique borrowed from the output regulation theory to handle sensor disturbances, and give a novel mechanism of designing measurement feedback control laws. Second, for over three decades, the output regulation problem is formulated as a disturbance rejection and tracking problem. However, it can only handle plant and actuator disturbances but not sensor disturbances. This paper enriches the output regulation theory to have the capacity of handling all three 
kinds of disturbances.

The remaining sections are organized as follows. In Section II, we formulate the problem, and offer some preliminary results on the necessary conditions for sensor disturbance rejection. The main result is given in Section III where a paradigm is established to systematically convert the measurement feedback problem into a state feedback problem. Finally, in Section IV we close this paper with some concluding remarks.

\section{Problem Formulation And Preliminaries}

Consider a nonlinear system

$$
\dot{x}=f(x, u, w)
$$

where $x \in \mathbb{R}^{n}$ is the plant state, $u \in \mathbb{R}^{m}$ the plant input, and $w \in \mathbb{R}^{N}$ the unknown vector representing the plant disturbances, in particular, the parameter variations. The function $f$ is supposed to be locally Lipschitz in $(x, u, w)$ with $f(0,0, w)=0$. And the class of state feedback control laws are described by

$$
\begin{aligned}
u & =k(x, z) \\
\dot{z} & =f_{z}(x, z)
\end{aligned}
$$

where $z \in \mathbb{R}^{n_{z}}$ is the compensator state vector with $n_{z}$ to be specified later and the functions $k$ and $f_{z}$ are continuously differentiable in $(x, z)$ with $k(0,0)=0$ and $f_{z}(0,0)=0$. In particular, if $n_{z}=0$, (2) is called a static controller. However, controller (2) can only be implemented in the ideal environment in absence of sensor disturbances. Otherwise, the controller (2) is implemented as

$$
\begin{aligned}
u & =k(\hat{x}, z) \\
\dot{z} & =f_{z}(\hat{x}, z)
\end{aligned}
$$

where $\hat{x}:=x+d$ is the measurement state and $d(t)$ represents the sensor disturbances. Throughout this paper, (3) is called a measurement feedback controller. With the controller (3), the closed-loop system with state $x_{c}:=\operatorname{col}(x, z)$ can be written as

$$
\dot{x}_{c}=\left[\begin{array}{c}
f(x, k(x+d, z), w) \\
f_{z}(x+d, z)
\end{array}\right] .
$$

To achieve the aforementioned objective, it is nature to consider the robust stabilization problem. The robust stabilization problem in this paper is precisely defined as follows, which addresses the robustness property of the controller with respect to sensor disturbances.

Definition 2.1: Global Robust Stabilization Problem (GRSP) via Measurement Feedback: For plant (1), to design a controller (3) (or, functions $k$ and $f_{z}$ ) such that the equilibrium point $x_{c}=0$ of the closed-loop system (4) is globally robustly asymptotically stable for all disturbances $w$ and $d$ in the specified sets.

Remark 2.1: In the above definition, if the closed-loop system is locally robustly asymptotically stable for all disturbances $w$ and $d$ in the specified sufficiently small sets, then the controller (3) solves the measurement feedback (local) robust stabilization problem (LRSP).
In the above problem formulation, we do not study all disturbances, but those disturbances in the specified sets. This is because, roughly, it is almost impossible to design a controller to solve GRSP (or LRSP) with rejecting all sensor disturbances. We precisely state this claim in the following theorem, which gives the necessary conditions for GRSP (or LRSP) with sensor disturbance rejection. For the simplicity of presentation, we consider the case with $w \in \mathbb{R}^{0}$.

Theorem 2.1: (i) A controller (3) exists to solve the measurement feedback GRSP for any bounded continuously differentiable $d(t)$, only if the open loop system $\dot{x}=f(x, 0)$ is globally asymptotically stable.

(ii) For any given $\epsilon>0$, a controller (3) exists to solve the measurement feedback LRSP for any bounded continuously differentiable $d(t)\left(\sup _{t \geq 0}\|d(t)\| \leq \epsilon\right)$, only if the open loop system $\dot{x}=f(x, 0)$ is locally asymptotically stable.

Proof: (i) If such a controller exists, then the closed-loop system (4), rewritten by

$$
\dot{x}_{c}=\left[\begin{array}{c}
f(x, k(x+d, z)) \\
f_{z}(x+d, z)
\end{array}\right] .
$$

is globally robustly asymptotically stable, which implies there exists a class $\mathcal{K} \mathcal{L}$ function $\beta$, such that $x_{c}(t) \leq$ $\beta\left(\left\|x_{c}(0)\right\|, t\right)$. As a result, for any $x_{c}(0)=\operatorname{col}(x(0), z(0))$, there exists $R=\beta\left(x_{c}(0), 0\right)$, such that $\left\|x_{c}(t)\right\| \leq R, \forall t \geq$ 0 , for any bounded $d(t)$. In particular, choose $z(0)=0$, $d(0)=-x(0)$, and suppose $d(t)$ is produced by

$$
\dot{d}=-f(-d, 0) \text {. }
$$

Next, let $\bar{x}_{c}:=\operatorname{col}(\bar{x}, z)$ with $\bar{x}:=x+d$, we have

$$
\dot{\bar{x}}_{c}=\left[\begin{array}{c}
f(x, k(\bar{x}, z))-f(x-\bar{x}, 0) \\
f_{z}(\bar{x}, z)
\end{array}\right] .
$$

Clearly, $\bar{x}_{c}=0$ is the equilibrium point of system (7). Since $\bar{x}_{c}(0)=0$, we have

$$
\bar{x}_{c}(0)(t)=0, z(t)=0, \forall t \geq 0,
$$

that is, $d(t)=-x(t), \forall t \geq 0$. From $\|d(t)\|=\|x(t)\| \leq$ $\left\|x_{c}(t)\right\| \leq R$, the disturbance $d(t)$ is bounded. Clearly, $d(t)$ is also continuously differentiable. Finally, using (8) in (5) gives that $\dot{x}=f(x, k(0,0))$, hence $\dot{x}=f(x, 0)$ is globally asymptotically stable.

(ii) Similarly, if such a controller exists, then the closedloop system (5) is locally robustly asymptotically stable. Then, for any $\epsilon>0$, there exists a sufficiently small $0<$ $\delta<\epsilon$, such that the trajectories (5) starting from $x_{c}(0)$ with $\left\|x_{c}(0)\right\| \leq \delta$ satisfies $\left\|x_{c}(t)\right\| \leq \epsilon, \forall t \geq 0$. Now, choose any $x(0)$ with $\|x(0)\| \leq \delta, z(0)=0, d(0)=-x(0)$, and suppose $d(t)$ is produced by (6). Similarly, we have (8), hence, $\|d(t)\|=\|x(t)\| \leq \delta<\epsilon$. Finally, using (8) in (5) gives $\dot{x}=f(x, 0)$ is locally asymptotically stable. I

Remark 2.2: Theorem 2.1 can also be explained geometrically. If there exists a controller suppressing all sensor disturbances, it suppresses those produced by (6) if bounded. Clearly, the manifold $\left\{\operatorname{col}\left(x_{c}, d\right) \mid x=-d, z=0\right\}$ is invariant for the composite system consisting of (5) and (6). 
And in this manifold, (5) reduces to $\dot{x}=f(x, 0)$, which should be GAS (or LAS) since (5) is by assumption.

Remark 2.3: In Theorem 2.1, a necessary condition for measurement feedback LRSP or GRSP is that the open loop system is locally asymptotically stable, even when the disturbances are sufficiently small and continuously differentiable. Moreover, if the sensor disturbances are bounded (not sufficiently small), then the necessary condition for GRSP with rejecting all sensor disturbances becomes that the open loop system is globally asymptotically stable. When we study the stabilization problem, these stability assumptions on the open loop system make the problem trivial, however, they are necessary. Therefore, when we ask for the solvability of the robust stabilization problem with sensor disturbance rejection, it is too much to reject all disturbances, and it is more pragmatic and more tractable to deal with the disturbances in some specified sets or with certain constrains. In this paper, the precise meaning of the specified sets will be given in the next section. Roughly, we suppose the sensor disturbances are some exogenous signals produced by an autonomous system called exosystem. This kind of disturbances are widely used in literatures, especially, in the formulation of output regulation problem.

In Remark 2.3, it has been pointed out that to make the stabilization problem tractable, we require the disturbances be in some specified sets because it is too much to handle all disturbances. On the other hand, in most situation, it is also too much to ask for the solvability of the stabilization problem. Instead, it is enough to seek that of the regulation problem for achieving our aforementioned objective. Specifically, when we adopt a dynamic controller, that is, $n_{z}>0$ in controller (3), it is required to drive both the plant state $x$ and the compensator state $z$ to the origin in the formulation of the robust stabilization problem. In practice, it is enough to drive $x$ to the origin while maintaining $z$ bounded. In this sense, the problem is called robust regulation problem, as defined below.

Definition 2.2: Global Robust Regulation Problem (GRRP) via Measurement Feedback: For plant (1), to design a controller (3) (or, functions $k$ and $f_{z}$ ) such that the states of the closed-loop system are bounded, and $\lim _{t \rightarrow \infty} x(t)=0$ for all initial states of the closed-loop system, and all disturbances $w$ and $d$ in the specified sets. I

Remark 2.4: In the above definition, if the states of the closed-loop system are bounded and $\lim _{t \rightarrow \infty} x(t)=0$ for sufficiently small initial states of the closed-loop system, and all disturbances $w$ and $d$ in the specified sufficiently small sets, then the controller (3) solves the measurement feedback (local) robust regulation problem (LRRP).

Remark 2.5: It does make sense to keep $z$ active (not vanish along time), because, in handling the sensor disturbances, the compensator $(z)$ is designed to estimate the disturbances. Specifically, we will see in the next section that the dynamics governing $z$ capture the fundamental properties of $d$. In this sense, if the disturbance $d$ is persistently exciting, the state $z$ is always active, even at the steady time (when the plant state approaches the origin.) More discussion is given in Remark 3.4.

\section{A PARAdigm For MEASUREMENT FEEDBACK DESIGN}

Before studying the method to suppress the plant and sensor disturbances, we first give the precise meaning of the specified sets. We assume $w \in \mathbb{W}$ with $\mathbb{W}$ a compact subset of $\mathbb{R}^{N}$, and $d(t) \in \mathbb{D}$ with $\mathbb{D}$ defined by

$$
\begin{array}{r}
\mathbb{D}=\left\{d(t):[0, \infty) \mapsto \mathbb{R}^{n} \mid d(t)=\mathbf{d}(v(t)),\right. \\
\left.\dot{v}=A_{1} v, v \in \mathbb{R}^{q}, v_{0} \in \mathbb{V}_{0}\right\}
\end{array}
$$

where $\mathbf{d}$ is a sufficiently smooth function satisfying $\mathbf{d}(0)=$ 0 , and $\mathbb{V}_{0}$ is a compact subset of $\mathbb{R}^{q}$. From the definition of the set $\mathbb{D}$, we note $d(t)$ is produced by an autonomous exosystem

$$
\dot{v}=A_{1} v,
$$

and we assume that this exosystem is neutrally stable, i.e., all eigenvalues of $A_{1}$ are simple with zero real parts. Clearly, under the neutrally stability condition, we have $v(t) \in \mathbb{V}, t \geq$ 0 for some compact subset $\mathbb{V}$ of $\mathbb{R}^{q}$ if $v_{0} \in \mathbb{V}_{0}$. When we study the local case, we are interested in the specified sufficiently small sets. That is, we assume $\mathbb{W}, \mathbb{V}_{0}$ (hence $\mathbb{V})$ is sufficiently small compact sets. The assumption of $d(t) \in \mathbb{D}$ gives the structural information of the sensor disturbances. This assumption is a typical setting in the output regulation theory [9].

\section{A. Auxiliary Disturbance Generator}

We have assumed that the sensor disturbances are generated by an autonomous exosystem. By using this structural information, we aim to design an observer to estimate these disturbances. For this purpose, we should find an auxiliary disturbance generator, which can capture the essential information of the disturbances, and has certain observability property.

Definition 3.1: Let $g: \mathbb{R}^{n} \mapsto \mathbb{R}^{l}$ be a mapping for some positive integer $1 \leq l \leq n$. The nonlinear system (1) and (9) is said to have an auxiliary disturbance generator with output $g(d)$ if there exists a triple $\{\theta, \alpha, \beta\}$, where $\theta: \mathbb{R}^{q} \mapsto \mathbb{R}^{s}$, $\alpha: \mathbb{R}^{s} \mapsto \mathbb{R}^{s}$, and $\beta: \mathbb{R}^{s} \mapsto \mathbb{R}^{l}$ for some integer $s$ are sufficiently smooth functions vanishing at the origin, such that, for all trajectories $v(t)$ of the exosystem,

$$
\begin{aligned}
\frac{d \theta(v)}{d t} & =\alpha(\theta(v)) \\
g(\mathbf{d}(v)) & =\beta(\theta(v)) .
\end{aligned}
$$

If, in addition, the linearization of the pair $(\beta(\theta), \alpha(\theta))$ at the origin is observable, then $\{\theta, \alpha, \beta\}$ is called a linearly observable auxiliary disturbance generator with output $g(d)$.

\section{I}

Remark 3.1: The concept of auxiliary disturbance generator is derived from the steady-state generator given in [10]. The steady-state generator is used to produce the steadystate state and steady-state input of a nonlinear system when these steady-state values are perturbed away from zero by 
exogenous disturbances or required to be away from zero to track some reference signals. But, in this paper, the steadystate state and steady-state input are not away from the zero, and the auxiliary disturbance generator is used to reproduce the disturbances. Although those disturbances have been assumed to be produced by exosystem (9), the auxiliary disturbance generator captures the essential information of the disturbances, and it can have some observability property. Such observability property makes it possible to construct an appropriate observer for the disturbances, and the observer is also called an internal model. The steady-state generator is closely related to the concept of system immersion introduced in [1], and the details can be found in [10].

Remark 3.2: The function $g$ can be an identity function, and more generally, it can be $g(d)=\mathcal{D}$ with $\mathcal{D}:=$ $\left[d_{i_{1}}, d_{i_{2}}, \cdots, d_{i_{\ell}}\right]^{\top}$ where $1 \leq i_{1}<i_{2}<\cdots<i_{\ell} \leq n$ for some integer $\ell$ satisfying $0 \leq \ell \leq n$. The introduction of such function is for the case when disturbances only appear in sensors for state $x_{i_{k}}, k=1, \cdots, \ell$. Without loss of generality, we can relabel the index to make $i_{k}=k+(n-\ell)$. For simplicity of presentation, we make the partitions $x:=$ $\operatorname{col}\left(\mathcal{X}_{1}, \mathcal{X}_{2}\right)$ and $\hat{x}:=\operatorname{col}\left(\hat{\mathcal{X}}_{1}, \hat{\mathcal{X}}_{2}\right)$ with $\mathcal{X}_{2}, \hat{\mathcal{X}}_{2} \in \mathbb{R}^{\ell}$. Clearly, by these partitions, we have $\mathcal{X}_{1}=\hat{\mathcal{X}}_{1}$ and $\hat{\mathcal{X}}_{2}=\mathcal{X}_{2}+\mathcal{D}$.

Next, we give the conditions for the existence of an appropriate auxiliary disturbance generator. Fortunately, the condition can be given similarly as that for the existence of the steady-state generator in [10]. As follows, we modify some concepts and a lemma for our need from [10].

Definition 3.2: Let $\pi(v(t))$ be trigonometric polynomial of $t$ or polynomial in $v(t)$. Let $P(\lambda)=\lambda^{r}-a_{1}-a_{2} \lambda-$ $\cdots-a_{r} \lambda^{r-1}$ be a monic polynomial in $\lambda . P(\lambda)$ is called a zeroing polynomial of $\pi(v)$ if, along all trajectories $v(t)$ of the exosystem,

$$
\frac{d^{r} \pi(v)}{d t^{r}}-a_{1} \pi(v)-a_{2} \frac{d \pi(v)}{d t}-\cdots-a_{r} \frac{d^{r-1} \pi(v)}{d t^{r-1}}=0
$$

$P(\lambda)$ is called a minimal zeroing polynomial of $\pi(v)$ if $P(\lambda)$ is a zeroing polynomial of $\pi(v)$ of least degree. Moreover, let $\pi_{i}(v(t)), i=1, \cdots, I$, for some positive integer $I$, be $I$ trigonometric polynomials of $t$ or polynomials in $v(t)$. They are called pairwise coprime if their minimal zeroing polynomials $P_{1}(\lambda), \cdots, P_{I}(\lambda)$ are pairwise coprime .

Lemma 3.1: Suppose, for $i=1, \cdots, \ell$, there exist pairwise coprime polynomials $\pi_{i}^{1}(v(t)), \cdots, \pi_{i}^{I_{i}}(v(t))$ with $r_{i}^{1}, \cdots, r_{i}^{I_{i}}$ being the degrees of their minimal zeroing polynomials $P_{i}^{1}(\lambda), \cdots, P_{i}^{I_{i}}(\lambda)$, and a sufficiently smooth function $\Gamma_{i}: \mathbb{R}^{r_{i}} \mapsto \mathbb{R}\left(r_{i}=r_{i}^{1}+\cdots+r_{i}^{I_{i}}\right)$ vanishing at the origin such that, for all trajectories $v(t)$ of the exosystem,

$$
\begin{array}{r}
g_{i}(\mathbf{d}(v))=\Gamma_{i}\left(\pi_{i}^{1}(v), \dot{\pi}_{i}^{1}(v), \cdots, \frac{d^{\left(r_{i}^{1}-1\right)} \pi_{i}^{1}(v)}{d t^{\left(r_{i}^{1}-1\right)}, \cdots,}\right. \\
\left.\pi_{i}^{I_{i}}(v), \dot{\pi}_{i}^{I_{i}}(v), \cdots, \frac{d^{\left(r_{i}^{I_{i}}-1\right)} \pi_{i}^{I_{i}}(v)}{d t^{\left(r_{i}^{I_{i}}-1\right)}}\right) .
\end{array}
$$

(i) For $i=1, \cdots, \ell, j=1, \cdots, I_{i}$, let

$$
\begin{aligned}
\theta_{i}^{j}(v) & =\operatorname{col}\left(\pi_{i}^{j}(v), \dot{\pi}_{i}^{j}(v), \cdots, \frac{d^{\left(r_{i}^{j}-1\right)} \pi_{i}^{j}(v)}{d t^{\left(r_{i}^{j}-1\right)}}\right) \\
\theta_{i}(v) & =T_{i} \operatorname{col}\left(\theta_{i}^{1}(v), \cdots, \theta_{i}^{I_{i}}(v)\right)
\end{aligned}
$$

with $T_{i}$ being any nonsingular matrix of dimension $r_{i}, \Phi_{i}^{j}$ be the companion matrix of $P_{i}^{j}(\lambda)$ satisfying $\dot{\theta}_{i}^{j}(v)=\Phi_{i}^{j} \theta_{i}^{j}(v)$, $\Phi_{i}=$ block $\operatorname{diag}\left(\Phi_{i}^{1}, \cdots, \Phi_{i}^{I_{i}}\right)$, and $\beta_{i}\left(\theta_{i}\right)=\Gamma_{i}\left(T_{i}^{-1} \theta_{i}\right)$. Then the system composed of (1) and (9) has an auxiliary disturbance generator $\{\theta, \alpha, \beta\}$ with output $g(d)$ as follows

$$
\begin{aligned}
\theta(v) & =\operatorname{col}\left(\theta_{1}(v), \cdots, \theta_{\ell}(v)\right) \\
\alpha(\theta) & =T \Phi T^{-1} \theta \\
\beta(\theta) & =\operatorname{col}\left(\beta_{1}\left(\theta_{1}\right), \cdots, \beta_{\ell}\left(\theta_{\ell}\right)\right)
\end{aligned}
$$

where $\Phi=\operatorname{block} \operatorname{diag}\left(\Phi_{1}, \cdots, \Phi_{\ell}\right)$ and $T=$ block $\operatorname{diag}\left(T_{1}, \cdots, T_{\ell}\right)$.

(ii) For $i=1, \cdots, \ell$, let $\Psi_{i}=\left[\Psi_{i}^{1}, \cdots, \Psi_{i}^{I_{i}}\right]$ be the gradient of $\Gamma_{i}$ at the origin where $\Psi_{i}^{j} \in \mathbb{R}^{1 \times r_{i}^{j}}$. Then the pair $\left(\Psi_{i}, \Phi_{i}\right)$ is observable, hence, the generator (12) is linearly observable if

the pair $\left(\Psi_{i}^{j}, \Phi_{i}^{j}\right)$ is observable, $j=1, \cdots, I_{i}$.

I

\section{B. Internal Model}

Dynamics (10) describe the behavior of the disturbances, but this system is virtual, and its state $\theta$ cannot be measured. Now, a basic idea is to construct a dynamic compensator such that it captures the trajectory of (10), and its behavior at the steady time is exactly same as that governed by (10). This dynamic compensator is called an internal model candidate. If the state $\eta$ of the internal model candidate can be driven to asymptotically approach $\theta$ in certain sense by an appropriately designed controller, then the internal model candidate is also called an internal model. In the above sense, the internal model plays the role of observing the state of the disturbance generator. We also note that the concept of the internal model has been introduced for long history. Its meaning for linear robust output regulation problem has be precisely characterized [5], [6], and it has not been clearly defined for nonlinear systems even though it has been freely used in many papers by various authors. The meaning given here is closely related to that given in [10].

Definition 3.3: Suppose the nonlinear system (1) and (9) has an auxiliary disturbance generator with output $\mathcal{D}$. Let $\gamma$ : $\mathbb{R}^{s+n+m} \mapsto \mathbb{R}^{s}$ be a sufficiently smooth function vanishing at the origin. Then we call the following system

$$
\dot{\eta}=\gamma\left(\eta, \mathcal{X}_{1}, \hat{\mathcal{X}}_{2}, u\right)
$$

an internal model (candidate) of (1) and (9) with output $\mathcal{D}$ if

$$
\gamma(\theta(v), 0, \mathcal{D}, 0)=\alpha(\theta(v))
$$

Then 
Remark 3.3: We note that (14) depends on $\left(\mathcal{X}_{1}, \hat{\mathcal{X}}_{2}, u\right)$, all of which can be used in feedback design. As a result, (14) is implementable to estimate the steady state of the disturbances. But, in many cases, the internal model can be designed independent of $\mathcal{X}_{1}$ or $u$, e.g.,

$$
\dot{\eta}=\gamma\left(\eta, \hat{\mathcal{X}}_{2}\right)
$$

I

It will be seen that if the system (1) and (9) has an auxiliary disturbance generator, then it will have a variety of internal model candidates. In fact, the auxiliary disturbance generator itself is an internal model candidate upon defining $\gamma\left(\eta, \hat{\mathcal{X}}_{2}\right)=\alpha(\eta)$. However, this particular internal model candidate is not interesting because there is no way to make the $\eta$ approach $\theta$ without any driving. Here we will give a specific class of internal model candidates as follows.

Lemma 3.2: Under the same assumptions as those of Lemma 3.1, for $i=1, \cdots, \ell$, there exist controllable pairs $M_{i} \in \mathbb{R}^{r_{i} \times r_{i}}$ and $N_{i} \in \mathbb{R}^{r_{i} \times 1}$ with $M_{i}$ Hurwitz, such that

$$
\dot{\eta}=\gamma\left(\eta, \hat{\mathcal{X}}_{2}\right)=M \eta+N\left(\hat{\mathcal{X}}_{2}-\beta(\eta)+\Psi T^{-1} \eta\right)
$$

is an internal model candidate of the system (1) and (9) with output $\mathcal{D}$ where

$$
\begin{array}{r}
M=\text { block } \operatorname{diag}\left(M_{1}, \cdots, M_{\ell}\right) \\
N=\text { block } \operatorname{diag}\left(N_{1}, \cdots, N_{\ell}\right) \\
T=\text { block } \operatorname{diag}\left(T_{1}, \cdots, T_{\ell}\right)
\end{array}
$$

with $T_{i} \in \mathbb{R}^{r_{i} \times r_{i}}$ some nonsingular matrix satisfying the Sylvester equation

$$
T_{i} \Phi_{i}-M_{i} T_{i}=N_{i} \Psi_{i} .
$$

Proof: Since, for each $i=1, \cdots, \ell$, the spectra of the matrices $\Phi_{i}$ and $M_{i}$ are disjoint, and the pair $\left(\Psi_{i}, \Phi_{i}\right)$ is observable, there exists a unique, nonsingular matrix $T_{i}$, satisfying the Sylvester equation (18). From

$$
\begin{aligned}
& \gamma(\theta, \mathcal{D}) \\
= & M \theta+N\left(\mathcal{D}-\beta(\theta)+\Psi T^{-1} \theta\right) \\
= & M \theta+N \Psi T^{-1} \theta=T \Phi T^{-1} \theta,
\end{aligned}
$$

system (17) is an internal model candidate of (1) and (9) with output $\mathcal{D}$.

\section{Augmented Error System}

Attaching the internal model to the given plant yields the following augmented system

$$
\begin{aligned}
\dot{x} & =f(x, u, w) \\
\dot{\eta} & =\gamma\left(\eta, \hat{\mathcal{X}}_{2}\right) .
\end{aligned}
$$

Performing on (20) the following coordinate transformation

$$
\begin{aligned}
\bar{\eta} & :=\eta-\theta(v) \\
\overline{\mathcal{X}}_{1} & :=\mathcal{X}_{1} \\
\overline{\mathcal{X}}_{2} & :=\hat{\mathcal{X}}_{2}-\beta(\eta)
\end{aligned}
$$

gives the error system denoted by

$$
\begin{aligned}
\dot{\bar{x}} & =\bar{f}(\bar{x}, \bar{\eta}, u, v, w) \\
\dot{\bar{\eta}} & =\bar{\gamma}(\bar{x}, \bar{\eta}, u, v, w)
\end{aligned}
$$

with $\bar{x}:=\operatorname{col}\left(\overline{\mathcal{X}}_{1}, \overline{\mathcal{X}}_{2}\right)$. Now, we have the following result.

Lemma 3.3: Suppose system (1) and (9) has a auxiliary disturbance generator with output $\mathcal{D}$ and an internal model candidate described by (14). Then the augmented system in the new coordinates described by (22) has the property that, for all trajectories $v(t)$ of the exosystem, and all $w \in \mathbb{R}^{N}$,

$$
\begin{aligned}
& \bar{f}(0,0,0, v, w)=0 \\
& \bar{\gamma}(0,0,0, v, w)=0 .
\end{aligned}
$$

Proof: From the definition of the internal model, and the fact the $x=0$ is the equilibrium of (1) when $u=$ 0 , we conclude that for any $w \in \mathbb{R}^{N}$, the hypersurface $\{\operatorname{col}(x, \eta, v) \mid x=0, \eta=\theta(v)\}$ is an invariant submanifold of the composite system consisting of (20) and the exosystem (9) under the trivial feedback control $u=0$. Thus, under the state and input translation (21), this submanifold is represented by $\{\operatorname{col}(\bar{x}, \bar{\eta}, v) \mid \bar{x}=0, \bar{\eta}=0\}$ with the feedback control given by $u=0$. This is to say that the origin $(\bar{x}, \bar{\eta})=(0,0)$ is the equilibrium point of the unforced augmented system for all trajectories $v(t)$ of the exosystem, and any $w \in \mathbb{R}^{N}$. Thus the proof is completed.

\section{Solvability of LRRP/GRRP via Measurement Feedback}

Consider a controller of the form

$$
\begin{aligned}
u & =k(\bar{x}, \xi) \\
\dot{\xi} & =\zeta(\bar{x}, \xi)
\end{aligned}
$$

where $\xi \in \mathbb{R}^{n_{\xi}}$, and $k$ and $\zeta$ are sufficiently smooth satisfying $k(0, \cdots, 0)=0$, and $\zeta(0, \cdots, 0)=0$, and a corresponding measurement feedback controller of the form

$$
\begin{aligned}
u & =k\left(\mathcal{X}_{1}, \hat{\mathcal{X}}_{2}-\beta(\eta), \xi\right) \\
\dot{\eta} & =\gamma\left(\eta, \hat{\mathcal{X}}_{2}\right) \\
\dot{\xi} & =\zeta\left(\mathcal{X}_{1}, \hat{\mathcal{X}}_{2}-\beta(\eta), \xi\right) .
\end{aligned}
$$

Then, we have the following result.

Theorem 3.1: (i) If the controller (24) is such that the closed-loop system composed of (22) and (24) has a Hurwitz Jacobian matrix with $v(t)$ and $w$ being set to zero, then the controller (25) solves the measurement feedback LRRP for the original system (1).

(ii) If the controller (24) is such that the equilibrium $\operatorname{col}(\bar{x}, \bar{\eta}, \xi)=0$ of the closed-loop system composed of (22) and (24) is globally asymptotically stable for all $v(t) \in \mathbb{V}$ and all $w \in \mathbb{W}$, then the controller (25) solves the measurement feedback GRRP for the original system (1).

Proof: Denote the state of the closed-loop system composed of the plant (1) and the controller (25) by $x_{c}:=$ $\operatorname{col}(x, \eta, \xi)$, and that of the closed-loop system composed of the plant (22) and the controller (24) by $\bar{x}_{c}:=\operatorname{col}(\bar{x}, \bar{\eta}, \xi)$, respectively. Then

$$
x_{c}=\bar{x}_{c}+\operatorname{col}(0, \beta(\bar{\eta}+\theta)-\beta(\theta), \theta, 0) .
$$


Part (i). By setting $v=0$ and $w=0$, the closed-loop system composed of (22) and (24) has a Hurwitz Jacobian matrix, then we have $\lim _{t \rightarrow \infty} \bar{x}_{c}(t)=0$ for all $v \in \mathbb{V}$ and $w \in \mathbb{W}$ with sufficiently small $\mathbb{V}$ and $\mathbb{W}$. Hence, we have $\lim _{t \rightarrow \infty} x(t)=0$ in the closed-loop system (1) and (25).

Part (ii). From (26), if the closed-loop system composed of (22) and (24) is globally asymptotically stable, then we have $x_{c}(t)$ starting from any initial state $x_{c}(0)$ exists and satisfies $\lim _{t \rightarrow \infty} \bar{x}_{c}(t)=0$ for all $v \in \mathbb{V}$ and $w \in \mathbb{W}$. By using the same argument as what is used in part (i), we can also conclude $\lim _{t \rightarrow \infty} x(t)=0$ in the closed-loop system composed of (1) and (25).

Remark 3.4: The controller (25) exactly takes the form of (3) with $z=\operatorname{col}(\eta, \xi)$. We note that $\eta$ essentially is the estimation of $\theta$, which represents the sensor disturbances. In other words, if the sensor disturbances persistently exist, so is $\theta$, then $\eta$ is always active to captures the fundamental information of the disturbances. That is the reason why we don't require $z$ approach zero at the steady time as pointed out in Remark 2.5.

Theorem 3.1 effectively concludes that a controller that solves the state feedback robust stabilization problem for system (22) also solves the measurement feedback robust regulation problem for system (1). Then, we can borrow the existing methods to solve the state feedback stabilization problem for a variety of nonlinear systems.

\section{CONCLUSION}

In this paper, we have developed a mechanism to reject sensor disturbances. A feature of the proposed control law is its ability of driving the plant state to the equilibrium point in presence of sensor disturbances. This mechanism has been applied to solve the global robust regulation problem of lower-triangular systems by partial measurement feedback control (see [2]). The mechanism is developed from the output regulation theory, and enriches the capacity of the output regulation theory to handle not only plant disturbances and actuator disturbances, but also sensor disturbances.

\section{REFERENCES}

[1] C. I. Byrnes, F. Delli Priscoli, A. Isidori, and W. Kang, "Structurally stable output regulation of nonlinear systems," Automatica, vol. 33, pp. 369-385, 1997.

[2] Z. Chen, "Global robust regulation of lower triangular systems via measurement feedback," IEEE International Conference on Control and Automation, 2007, to appear.

[3] Z. Chen and J. Huang, "Dissipativity, stabilization, and regulation of cascade-connected systems," IEEE Transactions on Automatic Control, vol. 49, pp. 635-650, 2004.

[4] Z. Chen and J. Huang, "A general formulation and solvability of the global robust output regulation problem," IEEE Transactions on Automatic Control, vol.50, pp. 448-462, 2005.

[5] E.J. Davison, "The robust control of a servomechanism problem for linear time-invariant multivariable systems," IEEE Transactions on Automatic Control, vol. 21, pp. 25 - 34, 1976.

[6] B.A. Francis and W.M. Wonham, "The internal model principle of control theory," Automatica, vol. 12, pp. 457-465, 1976.

[7] R.A. Freeman, "Global internal stabilization does not imply global external stabilibity for small sensor disturbances," IEEE Transactions on Automatic Control, vol. 40, pp. 2119-2122, 1995.
[8] R.A. Freeman and P.V. Kokotović, "Robust control Lyapunov functions: The measurement feedback case," Proceedings of the 33rd IEEE Conference on Decision and Control, Lake Buena Vista, FL, 1994, pp. 3533-3538.

[9] J. Huang, Nonlinear output regulation problem: Theory and Applications, SIAM, Philadelphia, 2004.

[10] J. Huang and Z. Chen, "A general framework for tackling the output regulation problem," IEEE Transactions on Automatic Control, vol. 49, pp. 2203-2218, 2004.

[11] A. Isidori and C.I. Byrnes, "Output regulation of nonlinear systems," IEEE Transactions on Automatic Control, vol. 35, pp. 131-140, 1990.

[12] Z.-P. Jiang, I. Mareels, and D. Hill, "Robust control of uncertain nonlinear systems via measurement feedback," IEEE Transactions on Automatic Control, vol. 44, pp.807-812, 1999.

[13] Mazenc F, Praly L, Dayawansa W P. Global stabilization by output feedback: examples \& counterexamples Systems and Control Letters, vol. 23, pp.119-125, 1994.

[14] E.D. Sontag, "Input/output and state-space stability," New Trends in System Theory, Boston, MA:Birkhauser, 1991, pp. 684-691. 